\title{
Thermodynamic Bethe ansatz and form factors for the homogeneous sine-Gordon models [t
}

\author{
Andreas Fring \\ Institut für Theoretische Physik, Freie Universität Berlin, Arnimalle 14, \\ D-14195 Berlin
}

January, 2001

\begin{abstract}
We provide a brief characterization of the main features of the homogeneous sine-Gordon models and discuss a general construction principle for colour valued S-matrices, associated to a pair of simply laced Lie algebras, which contain the homogeneous sine-Gordon models as a subclass. We give a brief introduction to the thermodynamic Bethe ansatz and the form factor approach and discuss explicit solutions for both methods related to the homogeneous Sine-Gordon models and its generalization.
\end{abstract}

Keywords: bootstrap program, thermodynamic Bethe ansatz, form factors, correlation functions, local operators, homogeneous sine-Gordon models, unstable particles, parity breaking

\section{Introduction}

The work I want to report about [1, 2, 3, 4, 5, 6] is based on a collaboration with Olalla Castro-Alvaredo (Universidad de Santiago de Compostela), Christian Korff (Stony Brook) and Luis Miramontes (Universidad de Santiago de Compostela).

The completion of the entire bootstrap program [7] for integrable quantum field theories in $1+1$ space-time dimensions remains an open challenge for most concrete models. Roughly speaking "the program" can be divided into five distinct steps: i) the determination of the exact on-shell S-matrix, ii) the computation of closed formulae for the $n$ particle form factors, iii) the identification of the entire local operator content, iv) the computation of the related correlation functions and $\mathrm{v}$ ) various non-perturbative consistency checks, like the thermodynamic Bethe ansatz, which exploit the information provided by the related conformal field theories. Step v) may of course also be carried out by resorting to standard old fashioned perturbative computations for various quantities. However, this is slightly opposed to the main virtue and

* Talk held at the NATO Advanced Research Workshop "Integrable Structures of Exactly Solvable Two-Dimensional Models of Quantum Field Theory", in Kiev (Ukraine), September 25-30 (2000) 
appeal of this approach, which is its entirely non-perturbative nature in the coupling constant.

Recently we investigated [1, 2, 3, 4, 5, 6] a class of models, the homogeneous Sine-Gordon model (HSG) [8] and its generalization, for which the bootstrap program was completed to a large extend. In comparison with other models, describable by a well-defined Lagrangian and which have been studied so far in $1+1$ dimensions, the HSG-models possess two interesting novel features familiar from realistic $1+3$ dimensional theories. Namely, their spectrum contains unstable particles and parity invariance is broken. With regard to one of the motivations for the study of lower dimensional theories, namely to treat them as test laboratories for general concepts and ideas, these properties deserve further consideration. One should say that unstable particles have first been investigated in $1+1$ dimensions in the context of the so-called roaming (staircase) models [9] and parity breaking was previously observed for the Federbush models [10], in which the scattering matrix is however just a trivial constant, i.e. rapidity independent, phase.

Due to the limited amount of time and space I will focus in my account on the main findings, referring the reader to the original literature [1, 2, 3, 4, 5, 6] and [11, 12, 13] for more details and general background.

\section{The homogeneous sine-Gordon models}

I want to start by recalling the main features of the $G_{k}$-HSG-models [8] related to simply laced Lie algebras. The latter have been constructed as integrable perturbations of gauged WZNW-coset theories [14] of the form $G_{k} / U(1)^{\ell}$, where $G$ is a compact simple Lie group of rank $\ell$ and $k>1$ the level of the Kac-Moody algebra. The defining action of the HSG-models reads

$$
S_{\mathrm{HSG}}[g]=S_{\mathrm{WZNW}}[g]+\frac{m^{2}}{\pi \beta^{2}} \int d^{2} x\left\langle\Lambda_{+}, g(\vec{x})^{-1} \Lambda_{-} g(\vec{x})\right\rangle .
$$

Here $S_{\text {WZNW }}$ denotes the coset action, $\langle$,$\rangle the Killing form of G$ and $g(\vec{x})$ a group valued bosonic scalar field. $\Lambda_{ \pm}$are arbitrary semi-simple elements of the Cartan subalgebra associated with the maximal abelian torus $H \subset G$, which have to be chosen not orthogonal to any root of $G$. The parameters $m$ and $\beta^{2}=1 / k+O\left(1 / k^{2}\right)$ are the bare mass scale and the coupling constant, respectively.

The study of this theory from a classical point of view has been carried out already to a very large extend and constitutes meanwhile a subject in its own right. Here I just want to focus on the properties 
which makes it an interesting theory from the quantum field theoretical point of view. In fact, as will be argued in the next sections, from that standpoint, one could abandon the classical picture altogether, since it just provides complementary but not necessary information. This is of course the natural algebraic quantum field theorist's viewpoint.

The classical equations of motion of these models correspond to non-abelian affine Toda equations [8, 15], which are known to be classically integrable and admit soliton solutions. The integrability on the quantum level was established in [16] by the construction of non-trivial conserved charges.

The coset conformal field theory defined by $S_{\mathrm{WZNW}}[g]$ is characterized by data which are in principle extractable from the thermodynamic Bethe ansatz (TBA) and the form factor analysis, namely the Virasoro central charge $c$ of the coset and the conformal dimensions $\Delta$ of various primary fields 14

$$
c_{G_{k} / U(1)^{\ell}}=\frac{k-1}{k+h} h \ell, \quad \Delta(\Lambda, \lambda)=\frac{(\Lambda \cdot(\Lambda+2 \rho))}{2(k+h)}-\frac{(\lambda \cdot \lambda)}{2 k} .
$$

Here $h$ is the Coxeter number, $\rho$ the Weyl vector, i.e. the sum over all fundamental weights, $\Lambda$ a highest dominant weight of level smaller or equal to $k$ and $\lambda$ their corresponding lower weights obtained in the usual way by subtracting multiples of simple roots $\alpha_{i}$ from $\Lambda$ until the lowest weight is reached. The specific choice of the groups ensures that these theories possess a mass gap [16]. Of particular interest is the dimension of the perturbing operator, that is the second term in (11), which is associated to the conformal dimension related to the adjoint representation $\Delta=\psi \equiv$ the longest root of $G$. We obtained the unique dimension $\Delta(\psi, 0)=h /(k+h)$. Since $\Delta(\psi, 0)<1$ for all allowed values of $k$, this perturbation is always relevant in the sense of renormalisation. To compute the conformal dimensions $\Delta(\Lambda, \lambda)$ of the entire primary field content is in general quite a formidable task. We report here only the $S U(3)_{2} / U(1)^{2}$ example with $c=6 / 5$, which we treat in detail in several different approaches. We obtained 四 12 fields with dimension $1 / 10,6$ fields of dimension $1 / 2,0$ and one field, i.e. the perturbing operator of dimension $\Delta(\psi, 0)=3 / 5$.

Having established that the theory defined by (1) constitutes an integrable massive quantum field theory suggests the existence of a factorizable scattering matrices which can be associated to it. Indeed, based on the assumption that the semi-classical spectrum is exact, the S-matrix elements have been conjectured in [17] and verified by means of the bootstrap principle for HSG-models related to simply laced Lie algebras. Instead of just stating these results, I would like to provide a 
slightly broader context and present a recently found type of scattering matrix of which the one of [17] is a special case.

\section{Colour valued S-matrices}

For this purpose I want to recall briefly the key features of step i) of the bootstrap approach. The fundamental observation, on which all further analysis relies upon, is that integrability, that means here the existence, one does not need to know its explicit form, of at least one non-trivial conserved charge, in $1+1$ space-time dimensions implies the factorization of the n-particle scattering matrix into a product of two particle scattering matrices

$$
Z_{\mu_{n}}\left(\theta_{n}\right) \ldots Z_{\mu_{1}}\left(\theta_{1}\right)|0\rangle_{\text {out }}=\prod_{1 \leq i<j \leq n} S_{\mu_{i} \mu_{j}}\left(\theta_{i j}\right) Z_{\mu_{1}}\left(\theta_{1}\right) \ldots Z_{\mu_{n}}\left(\theta_{n}\right)|0\rangle_{\text {in }} .
$$

It is usually convenient to parameterize the two-momentum $\vec{p}$ by the rapidity $\theta$ as $\vec{p}=m(\cosh \theta, \sinh \theta)$. We abbreviate $\theta_{A B}:=\theta_{A}-\theta_{B}$. The $Z_{\mu}(\theta)$ are creation operators for stable particles of type $\mu$ with rapidity $\theta$, which obey the Zamolodchikov algebra. The basic assumption of the bootstrap program is now that every solution to the unitary-analyticity, crossing and fusing bootstrap equations"

$$
S_{A B}(\theta)=S_{B A}(-\theta)^{-1}=S_{B \bar{A}}(i \pi-\theta), \quad \prod_{l=A, B, C} S_{D l}\left(\theta+i \pi \eta_{l}\right)=1,
$$

$\left(\eta_{l} \in \mathbb{Q}\right.$ are the fusing angles encoding the mass spectrum and the antiparticle of $A$ is $\bar{A}$ ), which admits a consistent explanation of all poles inside the physical sheet (that is $0<\operatorname{Im} \theta<\pi$ ), leads to a local quantum field theory. There exists no rigorous proof for this assumption, however, it is supported by numerous explicitly constructed examples. We may now exploit this principle and construct new solutions [2]. For this purpose we supply each particle type with an additional substructure and identify each particle by two quantum numbers, i.e. $A \equiv(a, i)$, such that the scattering matrices are of the form $S_{a b}^{i j}(\theta)$. We associate the main quantum numbers $a, b$ to the vertices of the Dynkin diagram of a simply laced Lie algebra $\mathbf{g}$ of rank $\ell$ and the colour quantum numbers $i, j$ to the vertices of the Dynkin diagram of a simply laced Lie algebra $\tilde{\mathrm{g}}$ of rank $\tilde{\ell}$. Noting now that for many (not always though) theories the scattering matrix factorizes into a so-called minimal part $S_{A B}^{\min }(\theta)$,

\footnotetext{
${ }^{1}$ For the purpose of this talk I suppose that there is no backscattering in the theory such that the Yang-Baxter equation constitutes no constraint.
} 
which satisfies (4) by itself, and into a CDD-factor $S_{A B}^{\mathrm{CDD}}(\theta, B(\beta))$ which depends on the effective coupling constant $B(\beta)$ containing only unphysical poles, $S_{A B}(\theta)=S_{A B}^{\min }(\theta) S_{A B}^{\mathrm{CDD}}(\theta, B(\beta))$, we may include the colour structure by defining the interaction as

$$
S_{a b}^{i j}(\theta)=\left\{\begin{array}{ll}
S_{a b}^{\min }(\theta)=\left(S_{a b}^{C D D}\left(\theta, B_{i i}=0\right)\right)^{-1} & \text { for } i=j \\
S_{a b}^{C D D}\left(\theta, B_{i j}\right) & \text { for } i \neq j
\end{array} .\right.
$$

Allowing now $\mathbf{g}, \tilde{\mathbf{g}}$ to be simply laced algebras, we found [2] as solution

$$
S_{a b}^{i j}(\theta)=e^{i \pi \varepsilon_{i j} K_{\bar{a} b}^{-1}+\int_{-\infty}^{\infty} \frac{d t}{t}\left(2 \cosh \frac{\pi t}{h}-\tilde{I}\right)_{i j}\left(2 \cosh \frac{\pi t}{h}-I\right)_{a b}^{-1} e^{-i t\left(\theta+\sigma_{i j}\right)} .}
$$

The theory possess various free parameters, the $\ell$ mass scales of the stable particles and the $\tilde{\ell}$ mass scales of the unstable particles characterized by the resonance parameters $\sigma_{i j}=-\sigma_{j i}(1 \leq i, j \leq \tilde{\ell})$ with the additional constraint that $i$ and $j$ are connected on the $\tilde{\mathbf{g}}$-Dynkin diagram.

As special cases of these models we have the $\mathbf{g} \mid \mathbf{A}_{1}$-theories and the $\mathbf{A}_{n} \mid \tilde{\mathbf{g}}$-theories, which coincide with the minimal affine Toda theories and the $\tilde{\mathbf{g}}_{n+1}$-HSG-models, respectively. S-matrices which allow the "colour algebra" $\tilde{\mathrm{g}}$ also to be non-simply laced were recently proposed in [18]. The generalization which also admits $\mathbf{g}$ to be non-simply laced still remains an interesting open problem. In that case the outlined factorization into minimal and CDD-factor does not take place anymore and one therefore has to abandon the described construction principle.

As already mentioned as particular example, which we want analyze in more detail, we consider the $\mathbf{A}_{1} \mid \mathbf{A}_{2} \equiv S U(3)_{2}$-HSG-model with

$$
S_{ \pm \pm}=-1 \quad, \quad S_{ \pm \mp}(\theta)= \pm \tanh (\theta \pm \sigma-i \pi / 2) / 2 .
$$

Since there is no pole in the physical sheet, fusing processes do not take place. An important point to note here, to which we frequently appeal, is that for the limit $\sigma \rightarrow \infty$ the S-matrices $\mathrm{S}_{ \pm \mp}$ tend to one and the whole theory decouples into a direct product of two thermally perturbed Ising models.

\section{Description of unstable particles}

Since the states in (3) are asymptotic, it is clear that unstable particles have to be described by different means, even when they possess a very long life time. As is familiar from standard quantum mechanics, one may introduce a decay width by complexifying the mass of a particle. As is for instance explained in [19], the same prescription can be taken 
over to quantum field theory. The description of an unstable particle of type $\tilde{c}$ may be thought of as adding a decay width $\Gamma_{\tilde{c}}$ to the physical mass of a stable particle, such that the S-matrix as a function of the Mandelstam $s$-variable has a pole at $s=M_{R}^{2}=\left(M_{\tilde{c}}-i \Gamma_{\tilde{c}} / 2\right)^{2}$. Transforming from the $s$ to the rapidity plane and describing the scattering of two stable particles of type $a$ and $b$ with masses $m_{a}$ and $m_{b}$ by $S_{a b}(\theta)$, the resonance pole is situated at $\theta_{R}=\sigma-i \bar{\sigma}$. Identifying the real and imaginary parts of the pole then yields

$$
\begin{aligned}
M_{\tilde{c}}^{2}-\Gamma_{\tilde{c}}^{2} / 4 & =m_{a}^{2}+m_{b}^{2}+2 m_{a} m_{b} \cosh \sigma \cos \bar{\sigma} \\
M_{\tilde{c}} \Gamma_{\tilde{c}} & =2 m_{a} m_{b} \sinh |\sigma| \sin \bar{\sigma} .
\end{aligned}
$$

Whenever $M_{\tilde{c}} \gg \Gamma_{\tilde{c}}$, the quantity $M_{\tilde{c}}$ admits a clear cut interpretation as the physical mass and the relations (8) and (9) acquire the form which is usually referred to as Breit-Wigner formula [20]. Since this assumption is only required for interpretational reasons we will not demand it in general. Eliminating the decay width from (8) and (9), we can express the mass of the unstable particles $M_{\tilde{c}}$ in the model as a function of the masses of the stable particles $m_{a}, m_{b}$ and the resonance parameter $\sigma$. Assuming $\sigma$ to be large we obtain

$$
M_{\tilde{c}}^{2} \sim m_{a} m_{b}(1+\cos \bar{\sigma}) / 2 e^{|\sigma|} .
$$

The occurrence of the variable $m e^{|\sigma| / 2}$ is interesting, since it was introduced originally in [21] in order to describe massless particles, i.e. one may perform safely the simultaneous limit $m \rightarrow 0, \sigma \rightarrow \infty$, and one might therefore be tempted to describe flows related to $(10)$ as so-called massless flows. In fact, in [1] we introduced the variable explicitly as a formal parameter.

One should note, however, a few essential differences between the roaming or staircase models [9] and the HSG models. First of all the decay width of the unstable particles enters in a different manner. Whereas in the HSG-model they are introduced by a rapidity shift $\theta \rightarrow \theta \pm \sigma$ of a parity broken theory, in the staircase models they enter through an analytic continuation at the self-dual point of the effective coupling constant $B$, i.e. $B \rightarrow 1 \pm i \sigma / \pi$, of scattering matrices associated to affine Toda field theories. Concerning the analytic continuation, the point $B=1$ is quite special, since for this point the combination $B(B-2)$ remains real. The other distinction between the two classes of models is the origin of the staircase pattern observed in the scaling functions of the models (see below). For the HSG-models one may associate the steps directly to the energy scale of the unstable particles, which is not possible for the staircase models. Furthermore, the HSG-models admit a well-defined Lagrangian. The condition $\Gamma_{\tilde{c}} \ll M_{\tilde{c}}$ corresponds to the semi-classical limit. 


\section{Thermodynamic Bethe Ansatz}

Originally the TBA was formulated in the context of the non-relativistic Bose gas by Yang and Yang [22] in order to extract various thermodynamic quantities. Thereafter it was extended [23] to relativistic quantum field theories whose scattering matrices factorize into twoparticle ones. In this context the approach allows the construction of a scaling function which reproduces some characteristic features, to be specified in more detail below, of the model and in particular various quantities of the underlying ultraviolet conformal field theory, in particular the effective Virasoro central charge and sometimes also the dimension of the perturbing operator.

It is well-known that in a relativistically invariant theory one can in general not use a wavefunction formalism, because of the creation of real and virtual particles. However, in configuration space one may assume to have regions in which one regards the particles as free and describe them formally by a function $\psi\left(x_{1}, \ldots, x_{N}\right), N$ being the total number of particles. Two adjacent free regions are then connected by an S-matrix. Taking now the particle $A$ on a trip around the world, a circle in this case, the formal wavefunction of $A$ picks up the corresponding S-matrix element as a phase factor when meeting another particle. For the case at hand we have to distinguish, whether the particle is moved clockwise or counter-clockwise along the world line, due to the breaking of parity invariance. Proceeding this way, we obtain two types of Bethe Ansatz equations

$$
e^{i L M_{A} \sinh \theta_{A}} \prod_{B \neq A} S_{A B}\left(\theta_{A B}\right)=e^{-i L M_{A} \sinh \theta_{A}} \prod_{B \neq A} S_{B A}\left(\theta_{B A}\right)=1,
$$

with $L$ denoting the length of the compactified space direction. These two sets of equations are of course not entirely independent and may be obtained from each other by complex conjugation and noting that $S$ is Hermitian-analytic [24]. Unlike as for instance in the talk of Slavnov (see this proceeding), we do not use the equations (11) to find allowed momenta, but instead we take the logarithm of these equations and carry out the thermodynamic limit in the usual fashion [23], i.e. letting the size of the system go to infinity $L \rightarrow \infty$, while keeping the ratio $L / N$ finite. This way we obtain the following sets of coupled non-linear integral equations

$$
\sum_{B} \Phi_{A B} * L_{B}^{ \pm}(\theta, r)=r M_{A} \cosh \theta+\ln \left(1-\exp \left(-L_{A}^{ \pm}(\theta, r)\right)\right) .
$$

Here the rapidity convolution of two functions is denoted by $f * g(\theta):=$ $\int d \theta^{\prime} / 2 \pi f\left(\theta-\theta^{\prime}\right) g\left(\theta^{\prime}\right)$. We also re-defined the masses by $M_{A} \rightarrow M_{A} / m_{1}$ 
keeping the same notation. The parameter $r=m_{1} T^{-1}$ is the inverse temperature times the overall mass scale of the lightest particle. The kernels in the integrals carry the information of the dynamical and statistical interaction of the system and are given by

$$
\Phi_{A B}(\theta)=\Phi_{B A}(-\theta)=-i \frac{d \ln S_{A B}(\theta)}{d \theta}-2 \pi g_{A B} \delta(\theta) .
$$

For generic values of $g_{A B}$ the statistical interaction is of Haldane type [25]. The special cases $g_{A B}=0$ and $g_{A B}=\delta_{A B}$ correspond to bosonic and fermionic statistics, respectively. Recently also a formulation for Gentile statistics has been proposed [26]. Here we will restrict our attention to the choice $g_{A B}=\delta_{A B}$. As very common in these considerations, we could also parameterize the function $L_{A}^{ \pm}(\theta)=\ln \left(1+e^{-\epsilon_{A}^{ \pm}(\theta)}\right)$ by the so-called pseudo-energies $\epsilon_{A}^{ \pm}(\theta)$. Then it follows from the properties of the TBA equations that $\epsilon_{A}^{+}(\theta)=\epsilon_{A}^{-}(-\theta)$. In comparison with the parity invariant case the main difference is that we have lost the usual symmetry of the pseudo-energies as a function of the rapidities, since we have $\epsilon_{A}^{+}(\theta) \neq \epsilon_{A}^{-}(\theta)$ in this case. The main task of the TBA is now to solve the equations (12) for the $L$ 's. Thereafter, one may compute the scaling function

$$
c(r)=\frac{3 r}{\pi^{2}} \sum_{A} M_{A} \int_{0}^{\infty} d \theta \cosh \theta\left(L_{A}^{-}(\theta)+L_{A}^{+}(\theta)\right),
$$

whose key property is

$$
\lim _{r \rightarrow 0} c(r)=c_{\mathrm{eff}}=c-24 \Delta^{\prime},
$$

with $c$ being the Virasoro central charge and $\Delta^{\prime}$ the lowest occurring conformal dimension of the underlying ultraviolet conformal field theory. We recall that for unitary models the values of $c$ and $c_{\text {eff }}$ coincide.

Due to the non-linear nature of the equations (12), there exist hardly any exact analytical solutions for the full TBA-equations, albeit it is possible to find various analytical approximations, e.g. [27]. When one is only interested in $c_{\text {eff }}$, it is possible to solve (12) and (14) fully analytically. Noting that the TBA-equations for the $\mathbf{g} \mid \tilde{\mathbf{g}}$-theories develop constant regions in this limit one may write (12) in this case in the very symmetric form

$$
\prod_{b=1}^{\ell}\left(Q_{b}^{i}\right)^{I_{a b}^{g}}+\prod_{j=1}^{\tilde{\ell}}\left(Q_{a}^{j}\right)^{I_{i j}^{\tilde{g}}}=\left(Q_{a}^{i}\right)^{2}
$$

Here we introduced the variable $Q_{a}^{i}=\prod_{b=1}^{\ell}\left(L_{b}^{i}\right)^{\left(K_{a b}^{\tilde{g}}\right)^{-1}}$. For finite values of the resonance parameter the effective central charge is then 
expressible as

$$
c_{\mathrm{eff}}^{\mathbf{g} \mid \tilde{\mathbf{g}}}=\frac{\ell \tilde{\ell} \tilde{h}}{h+\tilde{h}}=\frac{6}{\pi^{2}} \sum_{a=1}^{\ell} \sum_{i=1}^{\tilde{\ell}} \mathcal{L}\left(1-\prod_{b=1}^{\ell}\left(Q_{b}^{i}\right)^{-K_{a b}^{g}}\right)
$$

with $\mathcal{L}(x)=\sum_{n=1}^{\infty} x^{n} / n^{2}+\ln x \ln (1-x) / 2$ denoting Rogers dilogarithm. Remarkably, besides the numerical solutions, for many combinations of $\mathbf{g}$ and $\tilde{\mathbf{g}}$, the relations (16) have been solved analytically [28, 29, 1, 30] in such a way that the $Q_{a}^{i}$ 's acquire the form of certain Weyl-characters. Recalling that the $\mathbf{A}_{n} \mid \tilde{\mathbf{g}}$-theories $\equiv \tilde{\mathbf{g}}_{n+1}$-HSG-models we note that the first part of(2) and (17) obviously coincide.

From a purely mathematical point of view the system of equations (16) and second part of (17) is an exceptional situation whenever $c_{\text {eff }}$ is rational, in which case it is referred to as accessible dilogarithms (for a review see e.g. [31] and references therein). Remarkably, the same system of equations may be obtained by employing techniques originally pursuit in [32].
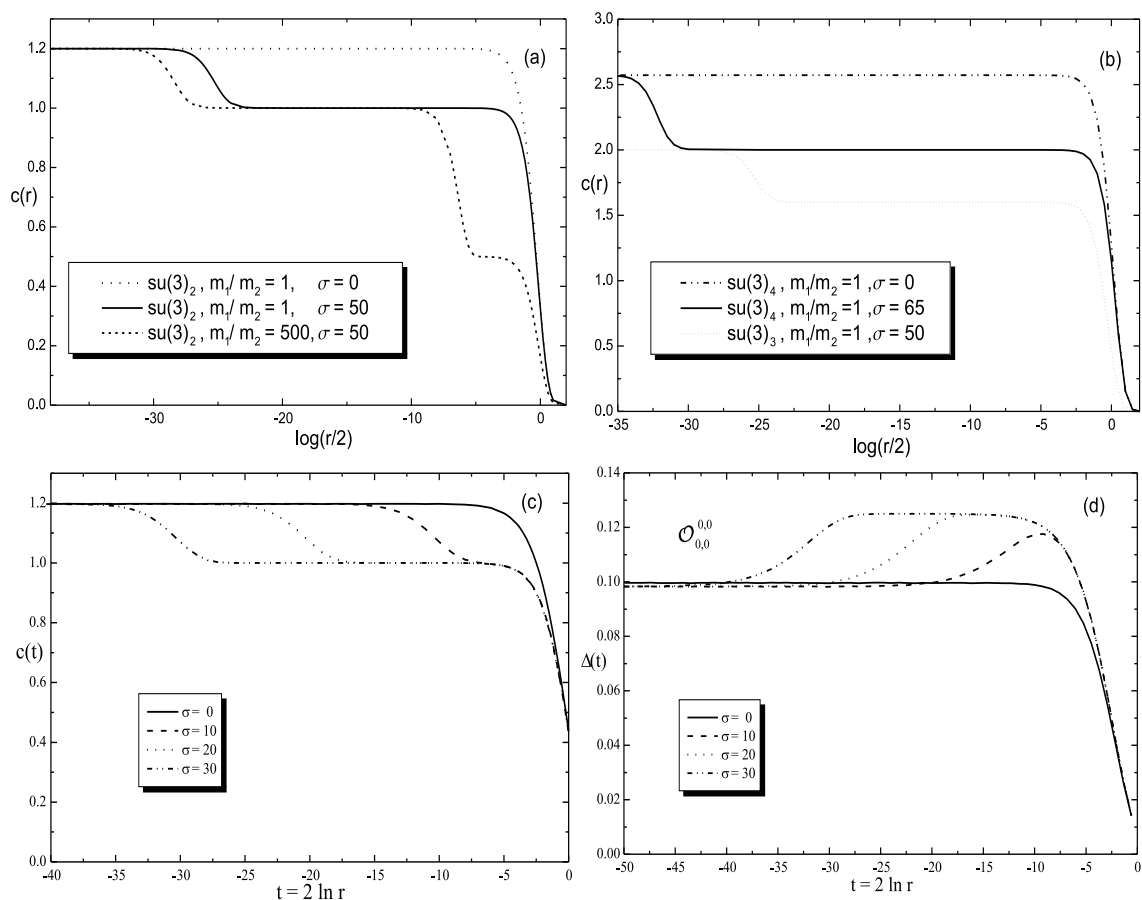

Figure 1: Scaling functions for $s u(3)_{k}, k=2,3,4$ from the thermodynamic Bethe ansatz as a function of the variable $\log r / 2$ with $r=m_{1} T^{-1}$ at different values of the resonance shift and mass ratios (a), (b). Scaling function from the correlation function $\langle\Theta(r) \Theta(0)\rangle$ for $s u(3)_{2}$ (c) and RG-flow of the conformal dimension of the local operator $\mathcal{O}_{0,0}^{0,0}$ (d) as a function of $2 \log r$. 
In [1] we analyzed the full TBA-equations for the $\mathbf{A}_{k-1} \mid \mathbf{A}_{2}$-theory $\equiv$ $\mathrm{SU}(3)_{k}$-HSG. The scaling function may be approximated in this case by

$$
c\left(r, M_{\tilde{c}}^{\tilde{k}}\right) \approx\left\{\begin{array}{ll}
4 \frac{k-1}{k+2}, & \text { for } \quad 1 \ll \frac{2}{r} \ll M_{\tilde{c}}^{\tilde{k}} \\
6 \frac{k-1}{k+3}, & \text { for } \quad M_{\tilde{c}}^{\tilde{k}} \ll \frac{2}{r}
\end{array},\right.
$$

where $M_{\tilde{c}}^{\tilde{k}}$ is the smallest mass of an unstable bound state which may be formed in the process $(a, i)+(b, j) \rightarrow(\tilde{c}, \tilde{k})$. This analytical approximation is confirmed by our full numerical solution as depicted in figure 1 (a), (b).

For the different algebras treated, we note that the different plateaus in the scaling functions are characterized by the mass scales of the unstable particles as described in (18). By choosing the masses of the two particles to be very different we also confirm in part (a) the decoupling of the theory into a direct product of two thermally perturbed Ising models. Exploiting the observation [1, 5, 6] that the plateaus are also realizable as cosets, it is also possible to find analytical descriptions similar in spirit to (16) and (17) for them [30.

\section{Form Factors}

To use form factors as auxiliary objects in order to compute correlation functions is an approach originally pioneered by the Berlin group in the late seventies [33]. Form factors are tensor valued functions, representing matrix elements of some local operator $\mathcal{O}(\vec{x})$ at the origin between a multiparticle in-state and the vacuum, which we denote by

$$
F_{n}^{\mathcal{O} \mid \mu_{1} \ldots \mu_{n}}\left(\theta_{1}, \ldots, \theta_{n}\right):=\left\langle 0|\mathcal{O}(0)| Z_{\mu_{1}}\left(\theta_{1}\right) Z_{\mu_{2}}\left(\theta_{2}\right) \ldots Z_{\mu_{n}}\left(\theta_{n}\right)\right\rangle_{\text {in }} .
$$

Here the $Z_{\mu}(\theta)$ 's are the same type of creation operators as in Eq. (3).

One way to determine the form factors, that is step ii) in the bootstrap program, is similar in spirit to the computation of the S-matrix, namely one considers the consequences various physical concepts have on these matrix elements. This way one can set up a system of constraints, which, like in the case of the scattering matrix, turns out to be so restrictive that they lead to their explicit determination. We shall

now only recall these constraints and refer for a more systematic and rigorous derivation of them to [34.

As a consequence of CPT-invariance or the exchange of two operators $Z_{\mu}(\theta)$ one obtains

$$
F_{n}^{\mathcal{O} \mid \ldots \mu_{i} \mu_{j} \ldots}\left(\ldots \theta_{i}, \theta_{j} \ldots\right)=F_{n}^{\mathcal{O} \mid \ldots \mu_{j} \mu_{i} \cdots}\left(\ldots \theta_{j}, \theta_{i} \ldots\right) S_{\mu_{i} \mu_{j}}\left(\theta_{i, j}\right) .
$$


The analytic continuation in the complex $\theta$-plane at the threshold cuts when $\theta=2 \pi i$ together with crossing leads to

$$
F_{n}^{\mathcal{O} \mid \mu_{1} \ldots \mu_{n}}\left(\theta_{1}+2 \pi i, \ldots, \theta_{n}\right)=F_{n}^{\mathcal{O} \mid \mu_{2} \ldots \mu_{n} \mu_{1}}\left(\theta_{2}, \ldots, \theta_{n}, \theta_{1}\right) .
$$

Since we are describing relativistically invariant theories, we expect for an operator $\mathcal{O}$ with $\operatorname{spin} s$

$$
F_{n}^{\mathcal{O} \mid \mu_{1} \ldots \mu_{n}}\left(\theta_{1}+\lambda, \ldots, \theta_{n}+\lambda\right)=e^{s \lambda} F_{n}^{\mathcal{O} \mid \mu_{1} \ldots \mu_{n}}\left(\theta_{1}, \ldots, \theta_{n}\right),
$$

with $\lambda$ being an arbitrary real number. For a form factor whose first two particles are conjugate to each other we have the so-called kinematical pole at $i \pi$, which leads to a recursive equation relating the (n-2)- and the n-particle form factor

$$
\begin{aligned}
-i \operatorname{Res}_{\bar{\theta}_{0} \rightarrow \theta_{0}} F_{n+2}^{\mathcal{O} \mid \bar{\mu} \mu \mu_{1} \ldots \mu_{n}}\left(\bar{\theta}_{0}+i \pi, \theta_{0}, \theta_{1}, \ldots, \theta_{n}\right)= \\
\quad\left(1-\omega \prod_{l=1}^{n} S_{\mu \mu_{l}}\left(\theta_{0 l}\right)\right) F_{n}^{\mathcal{O} \mid \mu_{1} \ldots \mu_{n}}\left(\theta_{1}, \ldots, \theta_{n}\right),
\end{aligned}
$$

with $\omega$ being the factor of local commutativity and $\bar{\mu}$ the anti-particle of $\mu$. We restrict our initial considerations to a model in which stable bound states may not be formed and therefore do not need to report on the so-called bound state residue equation, which relates an (n-1)and an n-particle form factor.

The equations (20)-(23) allow already the determination of several solutions. However, since these equations are completely independent of $\mathcal{O}(\vec{x})$, one needs further arguments to establish which type of operators one deals with. This means we are in a quite different position in comparison with the computation of matrix elements involving some well-known operator and Bethe states as described for instance in the talk by Kitanine (see this proceeding). There are however additional properties, which do involve the nature of the operator. In general they make use of their asymptotic behaviour, like the momentum space cluster property. It states that whenever the first, say $\kappa$, rapidities of an $n$-particle form factor are shifted to infinity, the $n$-particle form factor factorizes into a $\kappa$ and an $(n-\kappa)$-particle form factor, which are possibly related to different types of local operators

$$
\mathcal{T}_{1, \kappa}^{\lambda} F_{n}^{\mathcal{O}}\left(\theta_{1}, \ldots, \theta_{n}\right) \sim F_{\kappa}^{\mathcal{O}^{\prime}}\left(\theta_{1}, \ldots, \theta_{\kappa}\right) F_{n-\kappa}^{\mathcal{O}^{\prime \prime}}\left(\theta_{\kappa+1}, \ldots, \theta_{n}\right) .
$$

We have introduced here the operator $\mathcal{T}_{a, b}^{\lambda}:=\lim _{\lambda \rightarrow \infty} \prod_{p=a}^{b} T_{p}^{\lambda}$ which is composed of the translation operator $T_{a}^{\lambda}$ acting on a function of $n$ variables as $T_{a}^{\lambda} f\left(\theta_{1}, \ldots, \theta_{a}, \ldots, \theta_{n}\right) \mapsto f\left(\theta_{1}, \ldots, \theta_{a}+\lambda, \ldots, \theta_{n}\right)$. For the purely bosonic case this behaviour can be explained perturbatively 
by means of Weinberg's power counting theorem, see e.g. [33, 35]. For a similar restricted situation, there exists also a short heuristic argument which provides some form of intuitive picture of this behaviour [37] by appealing to the ultraviolet conformal theory. However, this argument is based on various so far unjustified assumptions, which need further clarification. In addition, up to now it has never been made use of. It is very interesting that when (24) is read from the r.h.s. to the l.h.s. it may been seen a kind of fusion ( $\neq$ the fusing mentioned in section 3 which refers to the particles) of the local operators. Therefore, in order to shed more light on step iii) of the bootstrap program, it would be important to put the property (24) on the same solid ground as the equations (20)-(23).

Having determined the form factors one may carry out step iv) of the bootstrap program and expand the two-point correlation function as

$$
\begin{aligned}
& \left\langle\mathcal{O}(r) \mathcal{O}^{\prime}(0)\right\rangle=\sum_{n=1}^{\infty} \sum_{\mu_{1} \ldots \mu_{n}} \int_{-\infty}^{\infty} \frac{d \theta_{1} \ldots d \theta_{n}}{n !(2 \pi)^{n}} e^{-r E} \\
& \times F_{n}^{\mathcal{O} \mid \mu_{1} \ldots \mu_{n}}\left(\theta_{1}, \ldots, \theta_{n}\right)\left(F_{n}^{\mathcal{O}^{\prime} \mid \mu_{1} \ldots \mu_{n}}\left(\theta_{1}, \ldots, \theta_{n}\right)\right)^{*}
\end{aligned}
$$

where $E=\sum_{i=1}^{n} m_{\mu_{i}} \cosh \theta_{i}$ is the sum of the on-shell energies. Provided we can compute (25) we may use the two-point correlation function in order to determine the RG-flow of the Virasoro central charge $c$ and the conformal dimension of a primary field. According to the c-theorem of Zamolodchikov we have

$$
\begin{aligned}
c(r)= & \frac{3}{2} \int_{r}^{\infty} d s s^{3}\langle\Theta(s) \Theta(0)\rangle \\
= & 3 \sum_{n=1}^{\infty} \sum_{\mu_{1} \ldots \mu_{n}} \int_{-\infty}^{\infty} \frac{d \theta_{1} \ldots d \theta_{n}}{n !(2 \pi)^{n}} e^{-r E} \\
& \times\left|F_{n}^{\Theta \mid \mu_{1} \ldots \mu_{n}}\left(\theta_{1}, \ldots, \theta_{n}\right)\right|^{2} \frac{\left(6+6 r E+3 r^{2} E^{2}+r^{3} E^{3}\right)}{2 E^{4}}
\end{aligned}
$$

where $\Theta$ is the trace of the energy momentum tensor. The flow of a conformal weight of a primary field was proposed in [5]

$$
\begin{aligned}
\Delta(r)= & -\frac{1}{2\langle\mathcal{O}(0)\rangle} \int_{r}^{\infty} d s s\langle\Theta(s) \mathcal{O}(0)\rangle \\
= & -\sum_{n=1}^{\infty} \sum_{\mu_{1} \ldots \mu_{n}} \int_{-\infty}^{\infty} \frac{d \theta_{1} \ldots d \theta_{n}}{n !(2 \pi)^{n}} \frac{(1+r E) e^{-r E}}{2\langle\mathcal{O}(0)\rangle E^{2}} \\
& \times F_{n}^{\Theta \mid \mu_{1} \ldots \mu_{n}}\left(\theta_{1}, \ldots, \theta_{n}\right)\left(F_{n}^{\mathcal{O} \mid \mu_{1} \ldots \mu_{n}}\left(\theta_{1}, \ldots, \theta_{n}\right)\right)^{*}
\end{aligned}
$$


as a straightforward generalization of the $\Delta$-sum rule [37]. The conformal dimension of a primary field may also be obtained by a direct analysis of the well-known ultraviolet behaviour of the correlation function

$$
\lim _{r \rightarrow 0}\langle\mathcal{O}(r) \mathcal{O}(0)\rangle \sim r^{-4 \Delta^{\mathcal{O}}}
$$

The main difference between the two possibilities is that (30) leads to far less conclusive values as (29). Whereas (30) requires in general an a priori knowledge of $\Delta^{\mathcal{O}}$, and serves mainly to confirm this, the expression (29) can just be evaluated by brute force.

I will now present the explicit solutions found for equations (20)(30) for the $S U(3)_{2}$-HSG model [3, 田, 5]. A step towards a more group theoretical understanding was done in [6], where these results were generalized to the $S U(N)_{2}$ case.

Labeling an operator by four quantum numbers $\mu, \nu, \tau, \tau^{\prime}$ we proved in [5] that the general n-particle solution reads

$$
\begin{aligned}
& F_{2 s+\tau, 2 t+\tau^{\prime}}^{\mathcal{O}_{\tau, \nu}^{\mu, \nu} \mid M^{+} M^{-}}\left(\theta_{1}, \ldots, \theta_{n}\right)=H_{2 s+\tau, 2 t+\tau^{\prime}}^{\mathcal{O}_{\tau, \nu}^{\mu, \nu} \mid M^{+}} \operatorname{det} \mathcal{A}_{2 s+\tau, 2 t+\tau^{\prime}}^{\mu, \nu} \\
& \left(\sigma_{2 s+\tau}^{+}\right)^{s-t+\frac{\tau-1-\nu}{2}}\left(\sigma_{2 t+\tau^{\prime}}^{-}\right)^{\frac{1+\tau-\tau^{\prime}-\mu}{2}-t} \prod_{i<j} \hat{F}^{\mu_{i} \mu_{j}}\left(\theta_{i j}\right) .
\end{aligned}
$$

W.l.g. we assumed here a particular ordering by starting with $2 s+\tau$ particles of the type $\mu=+$ followed by $2 s+\tau^{\prime}$ particles of the type $\mu=-$, collected in the sets $M^{ \pm}=\{ \pm, \ldots, \pm\}$. Once these expressions are known, all other form factors related to it by permutations of the particles may be constructed trivially by exploiting (20)-(21). The functions $\hat{F}^{\mu_{i} \mu_{j}}$ for all combinations of the $\mu$ 's are

$$
\begin{aligned}
& \hat{F}^{ \pm \pm}(\theta)=-i / 2 \tanh \frac{\theta}{2} \exp (\mp \theta / 2) \\
& \hat{F}^{ \pm \mp}(\theta)=2^{\frac{1}{4}} e^{\frac{i \pi(1 \mp 1) \pm \theta}{4}-\frac{G}{\pi}-\int_{0}^{\infty} \frac{d t}{t} \frac{\sin ^{2}\left((i \pi-\theta \mp \sigma) \frac{t}{2 \pi}\right)}{\sinh t \cosh t / 2},}
\end{aligned}
$$

with $G$ being the Catalan constant. The $(t+s) \times(t+s)$-matrix

$$
\left(\mathcal{A}_{2 s+\tau, 2 t+\tau^{\prime}}^{\mu, \nu}\right)_{i j}=\left\{\begin{array}{cl}
\sigma_{2(j-i)+\mu}^{+}, & 1 \leq i \leq t \\
\hat{\sigma}_{2(j-i)+2 t+\nu}^{-}, & t<i \leq s+t
\end{array}\right.
$$

has as its entries elementary symmetric polynomials depending on different sets of variables. We use the notation $\sigma^{ \pm}$when they depend on the variable $x=\exp \theta$ associated to the sets $M^{ \pm}$and $\hat{\sigma}$ to indicate that all variables are multiplied by a factor $i e^{-\sigma}$. The overall constant was computed to

$$
H_{2 s+\tau, 2 t+\tau^{\prime}}^{\mathcal{O}_{\tau, \tau^{\prime}}^{\mu, \nu}}=i^{s\left(2 \tau+\tau^{\prime}+\nu+2\right)} 2^{s\left(2 s-2 t-\tau^{\prime}-1+2 \tau\right)} e^{s \sigma\left(2 t+\tau^{\prime}\right) / 2} H_{\tau, 2 t+\tau^{\prime}}^{\mathcal{O}_{\tau, \tau^{\prime}}^{\mu, \nu}}
$$


where the value of $H_{\tau, 2 t+\tau^{\prime}}^{\mathcal{O}_{\tau, \nu}^{\mu, \nu}}$ is fixed by the lowest non-vanishing form factor. To determine this constant requires additional arguments. That these expressions are indeed solutions of (20)-(23) was rigorously proven in [5]. Alternatively we showed that the following cluster properties hold

$$
\begin{aligned}
\mathcal{T}_{1,2 \kappa+\xi \leq l}^{\lambda} F_{2 s+\tau, 2 t+\tau^{\prime}}^{\mu, 0} & \sim F_{2 \kappa+\xi, 0}^{0,0} F_{2 s+\tau-2 \kappa-\xi, 2 t+\tau^{\prime}}^{\mu+\xi(1-2 \mu), 0} \\
\mathcal{T}_{1,2 \kappa+\xi \leq l}^{-\lambda} F_{2 s+\tau, 2 t+\nu}^{\mu, \nu} & \sim F_{2 \kappa+\xi, 0}^{0,0} F_{2 s+\tau-2 \kappa-\xi, 2 t+\nu}^{\mu, \nu}, \\
\mathcal{T}_{n+1-2 \kappa-\xi<m, n}^{\lambda} F_{2 s+\tau, 2 t+\tau^{\prime}}^{0, \nu} & \sim F_{2 s+\tau, 2 t+\tau^{\prime}-2 \kappa-\xi}^{0, \nu+\xi(1-2 \nu)} F_{0,2 \kappa+\xi}^{0,0} \\
\mathcal{T}_{n+1-2 \kappa-\xi<m, n}^{-\lambda} F_{2 s+\mu, 2 t+\tau^{\prime}}^{\mu, \nu} & \sim F_{2 s+\mu, 2 t+\tau^{\prime}-2 \kappa-\xi}^{\mu, \nu} F_{0,2 \kappa+\xi}^{0,0},
\end{aligned}
$$

where we simplified the notation in an obvious way. The equations (36)(39) may be seen on one hand as a consistency check, since we recover the solutions constructed directly, and also as a construction principle, because from one particular solution we may derive various other ones, i.e. we simulate the "fusion" process.

Since the form factors of the trace of the energy-momentum tensor occur in various applications it is instructive to use it as an example to give the idea how more explicit formulae look like when all the quantities are assembled together

$$
F_{2 s, 2 t}^{\Theta}=\sigma_{1}\left(x_{1}, \ldots, x_{n}\right) \sigma_{1}\left(x_{1}^{-1}, \ldots, x_{n}^{-1}\right) F_{2 s, 2 t}^{\mathcal{O}_{2,2}^{1,1}}
$$

To fix the initial condition is in general also still a difficult issue, but we may solve this problem here easily by appealing to the fact that when the n-particle form factors involve only one type of particle they have to coincide with the ones for the thermally perturbed Ising model. The only non-vanishing form factor for the trace of the energy-momentum tensor is

$$
F_{2}^{\Theta}(\theta)=-2 \pi i m^{2} \sinh (\theta / 2)
$$

Explicit expressions for 4,6-particle form factors are for instance

$$
\begin{aligned}
F_{4}^{\Theta \mid++--} & =\frac{-\pi m_{-}^{2} e^{\left(\theta_{31}+\theta_{42}\right) / 2}\left(2+\sum_{i<j} \cosh \left(\theta_{i j}\right)\right)}{2 \cosh \left(\theta_{12} / 2\right) \cosh \left(\theta_{34} / 2\right)} \prod_{i<j} F_{\min }^{\mu_{i} \mu_{j}}\left(\theta_{i j}\right) \\
F_{6}^{\Theta \mid++++--} & =\frac{\pi m^{2}\left(3+\sum_{i<j} \cosh \left(\theta_{i j}\right)\right)}{4 \prod_{1 \leq i<j \leq 4} \cosh \left(\theta_{i j} / 2\right)} \prod_{i<j} \tilde{F}_{\min }^{\mu_{i} \mu_{j}}\left(\theta_{i j}\right) .
\end{aligned}
$$


The matrix $\mathcal{A}$ is in this case of dimension $(t+s-2) \times(t+s-2)$ and reads explicitly

$$
\mathcal{A}^{\Theta}=\left(\begin{array}{rrrrrr}
\sigma_{1}^{+} & \sigma_{3}^{+} & \sigma_{5}^{+} & \sigma_{7}^{+} & \cdots & 0 \\
0 & \sigma_{1}^{+} & \sigma_{3}^{+} & \sigma_{5}^{+} & \cdots & 0 \\
\vdots & \vdots & \vdots & \vdots & \ddots & \vdots \\
0 & 0 & 0 & 0 & \cdots & \sigma_{2 s-1}^{+} \\
-\hat{\sigma}_{1}^{-} & \hat{\sigma}_{3}^{-} & -\hat{\sigma}_{5}^{-} & \hat{\sigma}_{7}^{-} & \cdots & 0 \\
0 & -\hat{\sigma}_{1}^{-} & \hat{\sigma}_{3}^{-} & -\hat{\sigma}_{5}^{-} & \cdots & 0 \\
\vdots & \vdots & \vdots & \vdots & \ddots & \vdots \\
0 & 0 & 0 & 0 & \cdots & (-1)^{t} \hat{\sigma}_{2 t-1}^{-}
\end{array}\right) .
$$

Exploiting the integral representation for the symmetric polynomials $\sigma_{k}\left(x_{1}, \ldots, x_{n}\right)=\frac{1}{2 \pi i} \oint_{|z|=\varrho} \frac{d z}{z^{n-k+1}} \prod_{k=1}^{n}\left(z+x_{k}\right)$, it is easy to express the determinant of $\mathcal{A}^{\Theta}$ as a multidimensional integral representation

$$
\begin{gathered}
\operatorname{det} \mathcal{A}^{\Theta}=(-1)^{(s+1) t} \oint d u_{1} \ldots \oint d u_{t-1} \oint d v_{1} \ldots \oint d v_{s-1} \\
\times \prod_{j=1}^{t-1} u_{j}^{2-2 s-2 j} \prod_{i=1}^{2 s}\left(u_{j}+x_{i}\right) \prod_{j=1}^{s-1} v_{j}^{2-2 t-2 j} \prod_{i=1+2 s}^{2 s+2 t}\left(v_{j}+\hat{x}_{i}\right) \\
\quad \times \prod_{1 \leq i<j \leq t-1}\left(u_{j}^{2}-u_{i}^{2}\right) \prod_{1 \leq i<j \leq s-1}\left(v_{j}^{2}-v_{i}^{2}\right) \prod_{j=1}^{s-1} \prod_{i=1}^{t-1}\left(u_{i}^{2}+v_{j}^{2}\right)
\end{gathered}
$$

where we abbreviated $\oint d z \equiv(2 \pi i)^{-1} \oint_{|z|=\varrho} d z$ with $\varrho$ being a positive real number. These type of integrals differ from the ones provided in [34], where the integration is carried out in the $\theta$ rather than the $x$ variables. To manifest the precise relation between the two formulations remains an open issue at present.

Indicating by a superscript the number of the highest n-particle form factors taken into account in (27) and (29), we computed by means of a Monte Carlo integration

$$
\begin{aligned}
c(0)^{(2)} & =1, \quad c(0)^{(4)}=1.197 \ldots, \quad c(0)^{(6)}=1.199 \ldots, \quad \sigma<\infty \\
\lim _{\sigma \rightarrow \infty} c(0) & =1 .
\end{aligned}
$$

The latter equation is exact, since we have $\lim _{\sigma \rightarrow \infty} F_{2 s+2 t}^{\Theta \mid 2 s, 2 t} \sim e^{-(t+s-1) \sigma}$, from which we deduce that only $F_{2}^{\Theta \mid 0,2}$ and $F_{2}^{\Theta \mid 2,0}$ are non-vanishing, which allows an analytic evaluation. We obtained [ []

$$
\Delta^{\mathcal{O}_{0,0}^{0,0}(0)^{(6)}}=0.1004 \quad \sigma<\infty .
$$


The entire RG-flow of $c$ and $\Delta_{0,0}^{\mathcal{O}_{0,0}}$ is depicted in figure 1. We observe two crucial features: First of all, by comparing part (a) and (c) we see that the c-flow determined from (27) is qualitatively identical to the one obtained from the TBA. Secondly by comparing part (c) and (d) we observe that whenever the $S U(3)_{2}$-HSG model decouples into a direct product of two thermally perturbed Ising models, the $\mathcal{O}_{0,0}^{0,0}$-field decouples into two fields with conformal dimension 1/16, i.e. the disorder operator of the Ising model. We confirm these findings alternatively by a direct analysis of (30). A similar computation [4] was carried out also for several other solution (31). Unfortunately, we did not find solutions corresponding to all primary fields, in particular we did not find any solution corresponding to $\Delta=1 / 2$.

\section{Conclusions and open issues}

The main conclusion which can be drawn from our analysis concerning the specific status of the HSG-models ( $\mathbf{g} \mid \tilde{\mathbf{g}}$-theories) is that the scattering matrix originally proposed in [17] is certainly consistent and can be associated to a perturbed gauged WZNW-coset. This is based on the fact that we reproduce all the predicted features of this picture, namely the expected ultraviolet Virasoro central charge, various conformal dimensions of local operators and the characteristics of the unstable particle spectrum by means of the TBA as well as the form factor analysis.

The key statement concerning a comparison of the two different methods is that they yield the same qualitative behaviour of the scaling function. With regard to the efficiency of the methods one should point out that in the TBA approach the number of coupled non-linear integral equations to be solved increases with the number of different particles, which means the system becomes extremely complex and cumbersome to solve even numerically. Computing the scaling function with the help of form factors only adds more terms to each $n$-particle contribution, but is technically not more involved. The price we pay in this setting is, however, a slower convergence of (25). With regard to other quantities, that is the flow of the conformal dimensions, one should note that in the TBA-approach it is not known how to solve this problem and even at the fixed point itself one is, so far, only able to determine the conformal dimension of the perturbing operator with considerable effort for some particular theories. In the context of form factors there is no obstacle to the computation, once the solution is known.

Concerning the status of the bootstrap program, one should say that a very rich structure can be associated to step i). In particular, 
there exist closed general group theoretical expressions, which allow a relatively universal understanding as opposed to model dependent statements. Nonetheless. a complete classification is still outstanding. The status of step ii) is still less satisfactory since computable closed formulae exist only in few cases and one is still far from a closed group theoretical formulation. Step iii) also still requires some considerations, since up to now it is not even possible to identify properly the operators related to primary fields (we did not find various dimensions and can not undo the degeneracy), not to mention all the remaining operators. Further study of the cluster property will certainly provide advance in this direction. The computation of correlation functions, i.e. step iv) can in principle be carried out numerically, but of course a better analytical understanding is highly desirable. Our results [6] also indicate that the "folkloristic belief" of the fast convergence of the series expansion of (25) has to be challenged. In fact, for large values of $N$, this is not true anymore. It would be highly desirable to have more concrete quantitative criteria at hand. Needless to say that it would be nice to increase amount of technical tools available to carry out v) and enrich the spectrum of non-perturbative arguments.

\section{References}

1. O.A. Castro-Alvaredo, A. Fring, C. Korff and J.L. Miramontes, Nucl. Phys. B573 (2000) 535.

2. A. Fring and C. Korff, Phys. Lett. B477 (2000) 380.

3. O.A. Castro-Alvaredo, A. Fring and C. Korff, Phys. Lett. B484 (2000) 167.

4. O.A. Castro-Alvaredo and A. Fring, Identifying the Operator Content, the Homogeneous Sine-Gordon models hep-th/0008044.

5. O.A. Castro-Alvaredo and A. Fring, Phys. Rev. D63 (2001) 21701.

6. O.A. Castro-Alvaredo and A. Fring, Decoupling the $\mathrm{SU}(\mathrm{N})_{2}$-homogeneous Sine-Gordon model hep-th/0010262.

7. B. Schroer, T.T. Truong and P. Weisz, Phys. Lett. B63 (1976) 422; M. Karowski, H.J. Thun, T.T. Truong and P. Weisz, Phys. Lett. B67 (1977) 321; A.B. Zamolodchikov, JETP Lett. 25 (1977) 468.

8. Q-H. Park, Phys. Lett. B328 (1994) 329; T.J. Hollowood, J.L. Miramontes and Q-H. Park, Nucl. Phys. B445 (1995) 451; C.R. FernándezPousa, M.V. Gallas, T.J. Hollowood and J.L. Miramontes, Nucl. Phys. B484 (1997) 609.

9. Al.B. Zamolodchikov, Resonance Factorized Scattering and Roaming Trajectories, Preprint ENS-LPS-335 (1991).

10. P. Federbush, Phys. Rev. 121 (1961) 1247; B. Schroer, T.T. Truong and P. Weisz, Ann. Phys. 102 (1976) 156.

11. C. Korff, Lie algebraic structures in integrable models, affine Toda Field theory, PhD-Thesis (2000) hep-th/0008200. 
12. J.L. Miramontes, Integrable quantum field theories with unstable particles, Proceedings of the 4-th annual European TMR Conference on Integrability Nonperturbative Effects and Symmetry in Quantum Field Theory, Paris (2000) hep-th/0010012.

13. O.A. Castro-Alvaredo, PhD-Thesis (2001) in preparation.

14. E. Witten, Commun. Math. Phys. 92 (1984) 455; P. Goddard, A. Kent and D. Olive, Phys. Lett. B152 (1985) 88; V.A. Fateev and A.B. Zamolodchikov, Sov. Phys. JETP 62 (1985) 215; D. Gepner, Nucl. Phys. B290 (1987) 10; D. Gepner and Z. Qiu, Nucl. Phys. B285 (1987) 423.

15. A.N. Leznov and M.V. Saveliev, Commun. Math. Phys. 89 (1983) 59; L.A. Ferreira, J.L. Miramontes and J. Sánchez Guillén, Nucl. Phys. B449 (1995) 631.

16. C.R. Fernández-Pousa, M.V. Gallas, T.J. Hollowood and J.L. Miramontes, Nucl. Phys. B499 (1997) 673.

17. J.L. Miramontes and C.R. Fernández-Pousa, Phys. Lett. B472 (2000) 392.

18. C. Korff, Colours associated to non simply-laced Lie algebras and exact S-matrices, hep-th/0010287.

19. R.J. Eden, P.V. Landshoff, D.I. Olive and J.C. Polkinghorne, The analytic S-Matrix (CUP, Cambridge, 1966).

20. G. Breit and E.P. Wigner, Phys. Rev. 49, 519 (1936).

21. Al.B. Zamolodchikov, Nucl. Phys. B358 (1991) 524.

22. C.N. Yang and C.P. Yang, Phys. Rev. 147 (1966) 303; J. Math. Phys. 10 (1969) 1115.

23. Al.B. Zamolodchikov, Nucl. Phys. B342 (1990) 695; Nucl. Phys. B358 (1991) 497; Nucl. Phys. B366 (1991) 122.

24. D.I. Olive, Nuovo Cimento. 26 (1962) 73; G. Takács and G. Watts, Nucl. Phys. B547 (1999) 538; J.L. Miramontes, Phys. Lett. 455B (1999) 213.

25. A. Bytsko and A. Fring, Nucl. Phys. B532 [FS] (1998) 588.

26. A. Bytsko, Thermodynamic Bethe ansatz for generalized extensive statistics, hep-th/0011004.

27. A. Fring, C. Korff and B.J. Schulz, Nucl. Phys. B549 (1999) 579; A. Fring and C. Korff, Nucl. Phys. B579 [FS] (2000) 617.

28. A.N. Kirillov and N.Y. Reshetikhin, J. Sov. Math. 52 (1990) 3156.

29. A. Kuniba, Nucl. Phys. B389 (1993) 209; A. Kuniba and T. Nakanishi, Mod. Phys. 7 (1992) 3487; A. Kuniba, T. Nakanishi and J. Suzuki, Mod. Phys. 8 (1993) 1649; Int. J. Mod. Phys. 9 (1994) 5215; Int. J. Mod. Phys. 9 (1994) 5267.

30. O.A. Castro-Alvaredo and A. Fring, in preparation.

31. A.N. Kirillov, Prog.Theor.Phys.Suppl. 118 (1995) 61.

32. E.B. Richmond and G. Szekeres, J. Austral. Math. Soc. A31 (1981) 362.

33. P. Weisz, Phys. Lett. B67 (1977) 179; M. Karowski and P. Weisz, Nucl. Phys. B139 (1978) 445.

34. H. Babujian, A. Fring, M. Karowski and A. Zapletal, Nucl. Phys. B538 [FS] (1999) 535.

35. H. Babujian and M. Karowski, Phys. Lett. B471 (1999) 53.

36. A.B. Zamolodchikov, JETP Lett. 43 (1986) 730.

37. G. Delfino, P. Simonetti and J.L. Cardy, Phys. Lett. B387 (1996) 327. 